\title{
Localization and expression of EDS5H a homologue of the SA transporter EDS5
}

\author{
Nonglak Parinthawong ${ }^{1,2}$, Stéphanie Cottier ${ }^{1}$, Antony Buchala ${ }^{1}$, Christiane Nawrath ${ }^{1,3}$ and Jean-Pierre Métraux ${ }^{1 *}$
}

\begin{abstract}
Background: An important signal transduction pathway in plant defence depends on the accumulation of salicylic acid (SA). SA is produced in chloroplasts and the multidrug and toxin extrusion transporter ENHANCED DISEASE SUSCEPTIBILITY5 (EDS5; At4g39030) is necessary for the accumulation of SA after pathogen and abiotic stress. EDS5 is localized at the chloroplast and functions in transporting SA from the chloroplast to the cytoplasm. EDS5 has a homologue called EDS5H (EDS5 HOMOLOGUE; At2g21340) but its relationship to EDS5 has not been described and its function is not known.

Results: EDS5H exhibits about $72 \%$ similarity and $59 \%$ identity to EDS5. In contrast to EDS5 that is induced after pathogen inoculation, EDS5H was constitutively expressed in all green tissues, independently of pathogen infection. Both transporters are located at the envelope of the chloroplast, the compartment of SA biosynthesis. EDS5H is not involved with the accumulation of SA after inoculation with a pathogen or exposure to UV stress. A phylogenetic analysis supports the hypothesis that EDS5H may be an $\mathrm{H}^{+}$/organic acid antiporter like EDS5.

Conclusions: The data based on genetic and molecular studies indicate that EDS5H despite its homology to EDS5 does not contribute to pathogen-induced SA accumulation like EDS5. EDS5H most likely transports related substances such as for example phenolic acids, but unlikely SA.
\end{abstract}

\section{Introduction}

The signal transduction for induced resistance to many pathogens including viruses, bacteria, fungi and oomycetes involves the phenolic compound salicylic acid (SA) [1-3]. The importance of SA for the activation of defences has been repeatedly demonstrated with a number of mutants or transgenic plants impaired in the accumulation of SA. In particular, the Arabidopsis thaliana eds5/sid1 (enhanced disease susceptibility5/salicyclic acid-deficient 2) and sid 2 mutants accumulate only $10 \%$ of the SA produced in wild-type plants after induction and exhibit increased susceptibility to Pseudomonas syringae and Hyaloperonospora parasitica and fail to express the pathogenesis-related gene PR1 [4]. The eds5/sid 1 mutation was found in a gene encoding a member of the MATE (MULTIDRUG AND TOXIN EXTRUSION) transporter family [1], while the sid2 mutation was found in the ISOCHORISMATE SYNTHASE1 gene (ICS1) [5].

\footnotetext{
* Correspondence: jean-pierre.metraux@unifr.ch

'Department of Biology, University of Fribourg, 1700 Fribourg, Switzerland Full list of author information is available at the end of the article
}

The identification of ICS1 in A. thaliana, definitely demonstrated the importance of the isochorismate pathway for SA biosynthesis, similar to the pathway described in some Pseudomonas species [5]. The ICS1 gene product was confirmed to possess ICS activity and to be targeted to the plastidic compartment [6]. Synthesis of SA following exposure to ozone in Arabidopsis was also proposed to proceed through the activity of ICS enzymes [7]. The involvement of isochorismate in the synthesis of SA was confirmed in transgenic tobacco plants overexpressing an ICS of Catharanthus roseus [8] as well as in tomato [9] and Nicotiana benthamina [10]. The second ICS gene present in the Arabidopsis genome named ICS2 [5] encodes a protein that is also localized in the chloroplast and has ICS activity [11]. ICS2 participates in the synthesis of SA in partial redundancy with ICS1 since ics $1 /$ ics 2 double mutants produce only $36 \%$ of the SA amount found in the ics 1 single mutant. The conversion from isochorismate to SA has not yet been described in plants. In Pseudomonas aeruginosa it is catalyzed by a bifunctional enzyme displaying isochorismate pyruvate-lyase and chorismate mutase activities [12]. 
Thus, at the current state of knowledge, more than $95 \%$ of SA synthesized under inductive conditions is formed in the chloroplast.

Interestingly, a recent analysis showed that EDS5 is localized at the chloroplast envelope and functions in the export of SA from the chloroplast to the cytoplasm [13] [14]. It represents one of the few regulated transporters involved in the movement of a signal for induced defences.

In fact, MATE-transporters are present in almost all prokaryotes and eukaryotes and are thus one of the most conserved families in nature $[15,16]$. Plants have the largest gene family of MATE-transporters with 58 genes in Arabidopsis, while prokaryotes have approximately 10 genes and mammals only 2. Plant MATE-transporters have not only been found at the plasma membrane, but also at the vacuolar membrane acting in the sequestration of toxic metabolites. For example, the MATEtransporter TT12 acts as flavonoid $/ \mathrm{H}^{+}$antiporter at the vacuole and is active in proanthocyanidin accumulation in the seed coat of Arabidopsis [17, 18]. In tobacco, the alkaloid nicotine is sequestered into the vacuole in exchange with protons by the action of NtMATE1 and NtMATE2 in the roots and by the MATE-transporter NtJAT1 in the shoots $[19,20]$. These MATE-transporters may also transport other alkaloids, such as anabasine, hyoscyamine, scopolamine or berberine, but no flavonoids. Here we report a detailed characterization of EDS5H, a close homologue of the SA transporter EDS5.

\section{Results}

\section{Identification and characterization of EDS5H}

The analysis of the Arabidopsis genome revealed a homologue of EDS5 that is encoded by the gene At2g21340 and was therefore named EDS5H. The EDS5H gene was characterized by amplification of a $1680 \mathrm{bp}$ cDNA by reverse transcriptase-mediated polymerase chain reaction (RT-PCR) and subsequently sequenced. The analysis of the $E D H 5$ sequence confirmed that $E D S 5 H$ has an open reading frame (ORF) of $1680 \mathrm{bp}$ encoding for a protein of 559 amino acids. The genomic region of EDS5H consists of 14 exons and 13 introns based on the annotated Arabidopsis genome. The alignment between the predicted protein sequences of EDS5 and EDS5H showed an overall $72 \%$ similarity and $59 \%$ identity. However, the 100 aa at the $\mathrm{N}$-terminus showed less conservation (20\% identity) (Fig. 1).

\section{Expression of EDS5H}

The expression of $E D S 5 H$ was studied in leaves of plants inoculated with Pseudomonas syringae pv tomato avrRpt2. As indicated in Fig. 2, the expression shows no change in response to infection. The activity of the promoter of EDS5H was also studied using promoter-GUS fusions. Promoter fragments of 500, 1000 or $2000 \mathrm{bp}$ length were fused to GUS. Transgenic Arabidopsis plants carrying $p E D S 5 H:: G U S$ fusions of different lengths all gave the same activity pattern. The $E D S 5 H$ promoter was very active in all green tissues but not in roots or in petals (Fig. 3).

\section{Subcellular localization of EDS5H}

The ORF of $E D S 5 H$ was placed under the control of the CaMV 35S promoter upstream of a triple cMyc epitope tag. In tissue sections of transgenic plants carrying the CaMV35S::EDS5H::3xcMyc construct, the cMyc-epitope tag was labelled with a primary anti-myc antibody that was identified by a goat anti-mouse IgG conjugated with Alexa Fluor 488 (green). RCCR (red chlorophyll catalase reductase) was used as an example for a protein targeted to the chloroplast [21]. Tissues were labelled with a primary antibody against RCCR and secondary antibody goat anti-rabbit IgG conjugated with Alexa Fluor 568 (red). Fig. 4 shows an intense and fine green labelling localized around the chloroplast, while the red label was in the middle of the chloroplast. The co-localization of the EDS5H with RCCR shows that EDS5H is located to the chloroplast. cMyc-tag could not be detected in plant cells when the natural EDS5H promoter was used, although the transcription of the EDS5H:: cMyc could be determined by RT-PCR (data not shown). This suggests that the $E D S 5 H$ promoter, which is constitutive, was too weak to drive the whole cassette of the $E D S 5 H:: 3 \mathrm{xcMyc}$ to its target organelle or the amount was below detection limits. Taken together, these studies show that EDS5H is localized at the chloroplast as was shown previously for EDS5 [11].

\section{Analysis of plants with downregulated EDS5H expression}

Since both EDS5 and EDS5H are expressed in the same tissues and in the same organelle it is possible that EDS5H may also function in SA accumulation since it has been observed that eds5-3 mutants still contain ca $10 \%$ of the SA level found in wild-type plants [2]. This level of SA could potentially be due to the functional EDS5H. A mutant carrying a T-DNA insertion in the $E D S 5 H$ gene, called eds $5 h-1$, was isolated after screening the population of T-DNA insertion pools generated by the Arabidopsis knock-out facility service at the University of Wisconsin-Madison. Two mutant lines were obtained, eds5h-1 carrying the T-DNA insertion at 229 bp (exon 1) and eds5h-10 carrying the insertion at $1360 \mathrm{bp}$ (exon 10). Both were transcriptional null alleles, and the eds $5 h-1 \mathrm{mu}-$ tant was used for further studies. The expression of $E D S 5 H$ in Ws wild-type plants was strong in noninoculated leaves (Fig. 5a). The expression of EDS5 was not different between wild type and the eds5h-1 mutant (data not shown). No difference was detectable in the accumulation of SA after inoculation with $P$. syringae $p v$. 


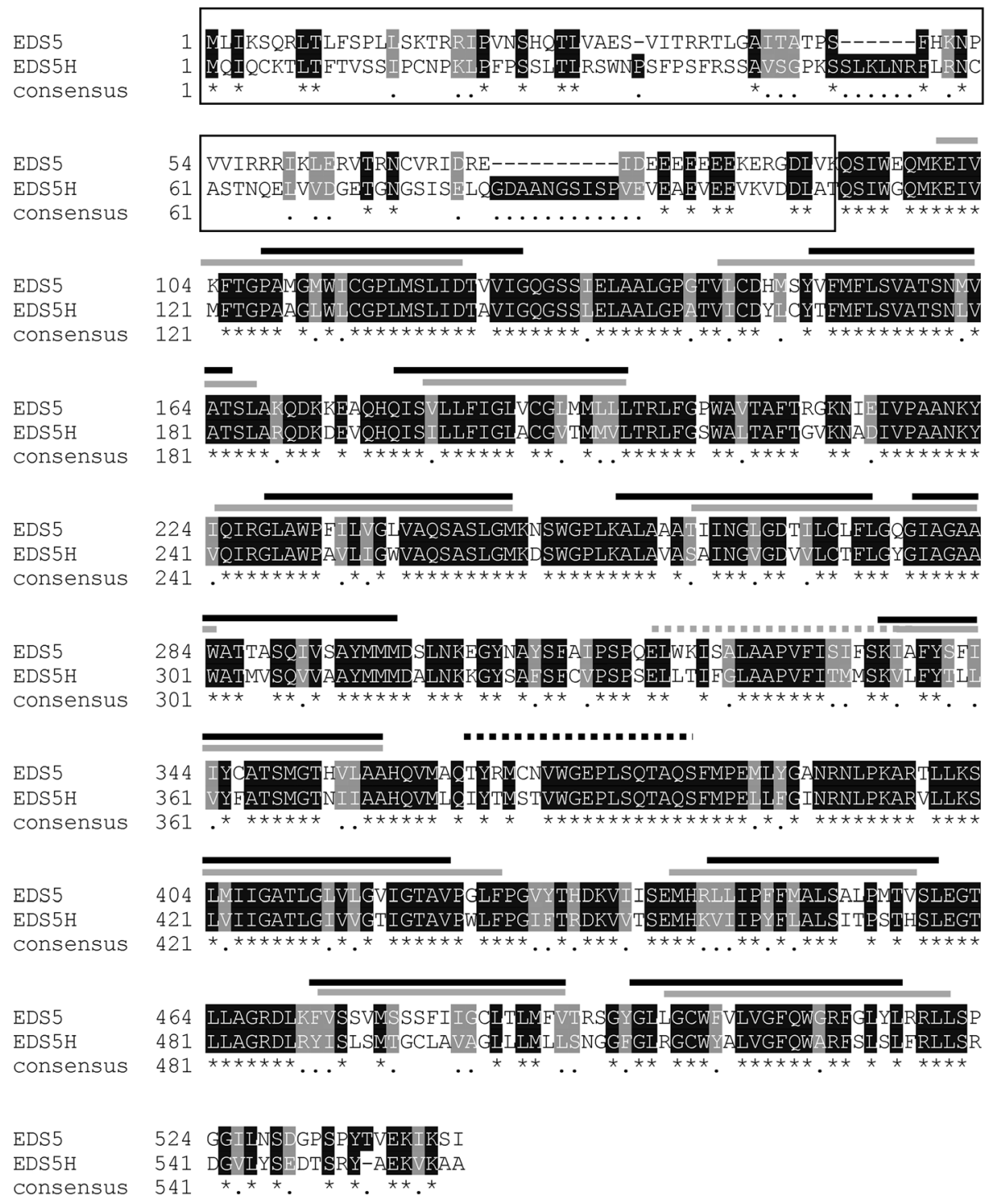

Fig. 1 Alignment of the predicted protein sequences of EDS5H and EDS5. The predicted membrane-spanning domains are indicated above the alignment with a grey bar for EDS5H and black bars for EDS5. Identical amino acids are indicated with an asterisk, and conserved amino acids are indicated with a single dot. The N-terminus region has a low degree of homology and is indicated by a black frame

tomato DC3000 in Ws and eds5h-1, most likely due to EDS5 function (Fig. 5b). Since the induction kinetics of SA accumulation in Col-0 and Ws showed strong differences, the generation of a double mutant between eds5h-1 (in the Ws background) and eds5-3 (in the Col-0 background) was not useful (data not shown). Therefore, we used RNA interference (RNAi) to downregulate the transcription of $E D S 5 H$ in the Col-0 background. For the construction of the RNAi construct, the 350 bp 5'-non-conserved region of $E D S 5 H$ was cloned into the plasmid pHANNIBAL [22] in sense and antisense orientations. The RNAi construct was transformed into both Col-0 and eds5-3. Independent lines from eds5h (EDS5H-RNAi) and from the double mutant eds5-3/eds5h (eds5-3/EDS5H-RNAi) were selected for further investigation because they did not accumulate EDS5H mRNA (Fig. 6a). These lines showed no changes in phenotype compared to wild-type plants. Transgenic plants in which no $E D S 5 H$ transcription was detected after infection with $P$. syringae $p v$. tomato DC3000 with or without the avirulence gene avrRpt2 were analysed for their SA content in comparison to Col-0 plants. A slight decrease in SA accumulation (ca $20 \%$ ) was observed in EDS5H-RNAi plants $12 \mathrm{~h}$ after infection with $P$. syringae pv. tomato DC3000 avrRpt2 (Avr) compared to Col-0 but this difference disappeared $24 \mathrm{~h}$ after infection. Similarly, no changes were observed when EDS5H was knocked 


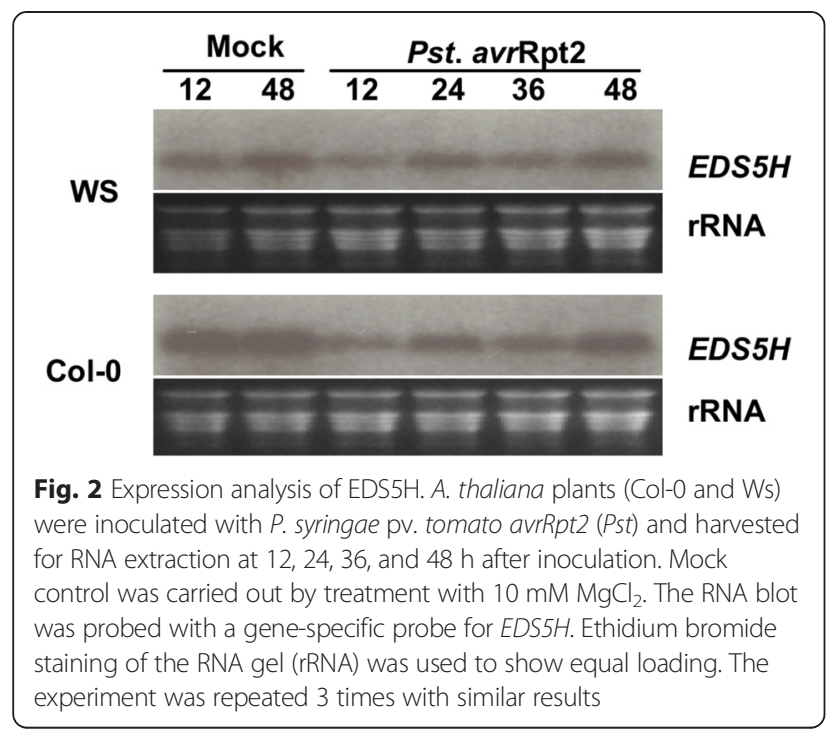

down by RNAi in the eds5-3 mutant (Fig. 6b). Similar results have been found for the SA accumulation after UVlight exposure (data not shown). These results support that $E D S 5 H$ is not responsible for the presence of the residual SA in the eds5-3 mutant.

\section{Analysis of transgenic eds5-3 plants overexpressing EDS5H and a EDS5::EDS5H fusion protein}

In order to determine if overexpression of $E D S 5 H$ can rescue the SA-deficient phenotype of eds5-3, EDS5H was expressed in eds5-3 under the control of the 35S promoter (Fig. 7a). The lines overexpressing EDS5H did not display any phenotypic change compared to wild types. Transgenic plants overexpressing $E D S 5 H$ were exposed to UVlight to induce the SA-biosynthesis pathway and their SA content was measured $12 \mathrm{~h}$ after induction. Some of the independent eds5-3 lines overexpressing EDS5H accumulated up to 3 times more SA than the eds5-3 controls but still 5 times less than the wild-type plants (Fig. 7b). The rescue of the eds5-3 mutant phenotype by the CaMV35S::EDS5H construct was therefore only limited.

EDS5 and EDS5H show a very high percentage of homology at the $\mathrm{C}$-terminal region containing the transmembrane spanning domains. In contrast, a small domain of approximately 60 aa that lies between the signal peptide for plastid targeting and the beginning of the transmembrane spanning domains shows little homology. In EDS5, this domain contains a hepta-peptide potentially forming a coiled-coil domain (http://embnet.vital-it.ch/software/COILS_form.html), while not in EDS5H. In order to test if the N-terminal domain confers specificity to the function of EDS5, eds5-3 plants were transformed with a CaMV35S::EDS5-EDS5H fusion that contained the plastid localization signal from EDS5 as well as the $\mathrm{N}$-terminus of the mature protein. The

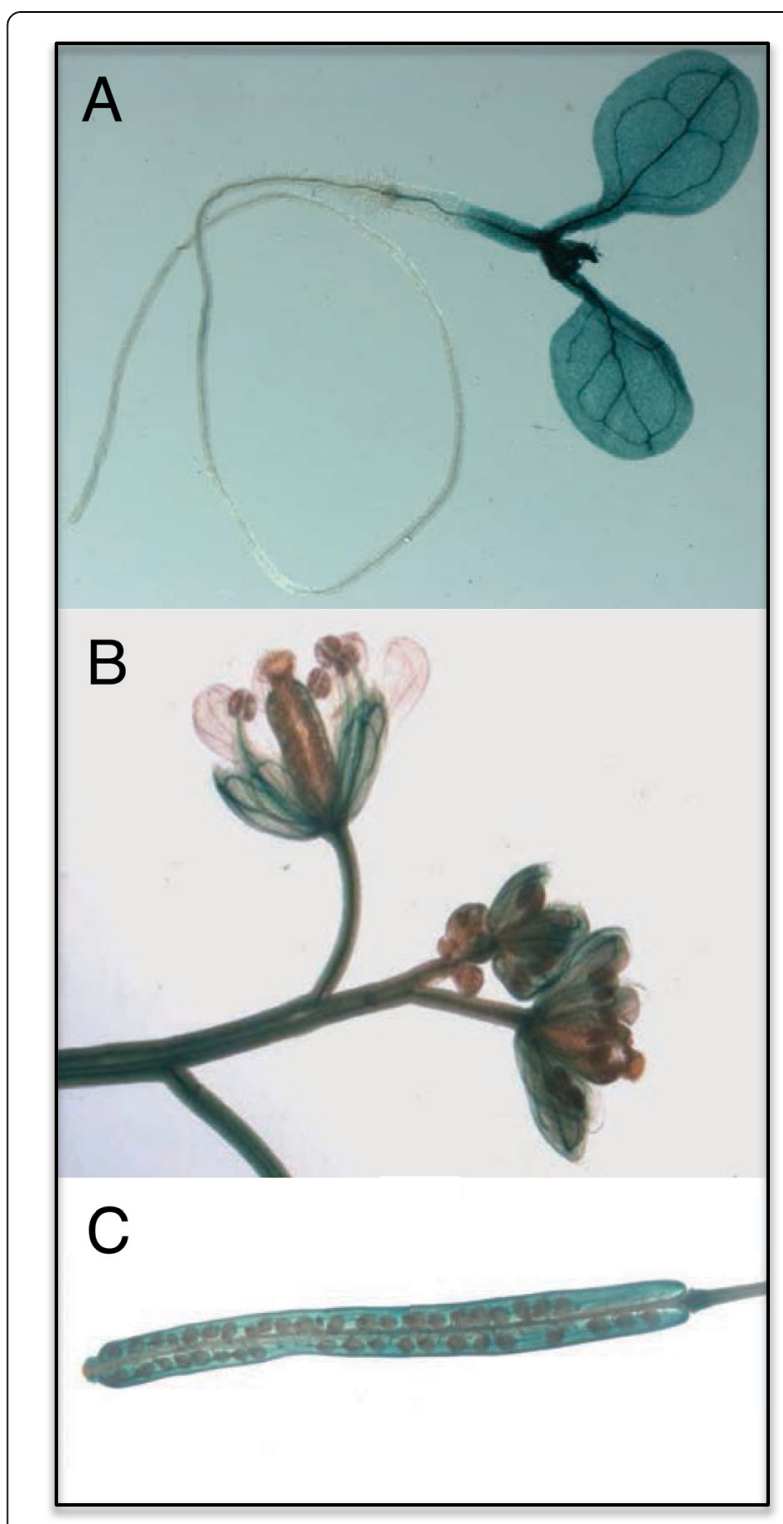

Fig. 3 Localization of GUS activity in transgenic Arabidopsis plants expressing the pEDS5H::GUS fusion construct. Seedlings, siliques or inflorescence parts of plants transformed with EDS5H::GUS constructs were stained with the substrate for GUS. The activity of a promoter of 2000 bp length is shown in a seedling (a), flowers (b), and a silique (c). The experiment was repeated 3 times with similar results

accumulation of SA was monitored in transgenic eds5-3 plants overexpressing the EDS5::EDS5H fusion protein after their exposure to UV-light followed by a 12-h incubation time. The expression of the EDS5::EDS5H fusion protein did not result in a better rescue of the eds5-3 phenotype than the original EDS5H (Fig. 7b), but still increased the SA amount of the eds 5 mutant suggesting that the resulting fusion protein still had transport activity. The fusion of the entire $\mathrm{N}$-terminal domain of 

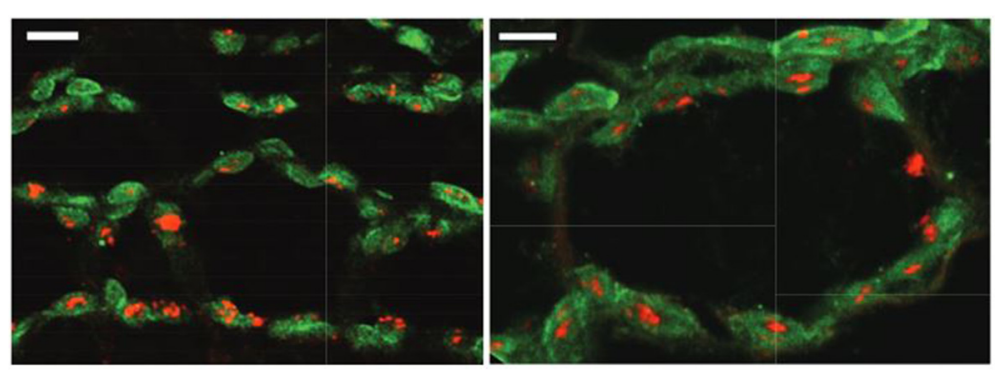

Fig. 4 Subcellular localization of EDS5H-3myc in transgenic Arabidopsis. Mesophyll cells of transgenic plants carrying the CaMV35S::EDS5H::3xmyc construct. The two panes show the cMyc-epitope labelled with Alexa Fluor 488 excited at the wavelength of $488 \mathrm{~nm}$ and detected using the emission filter 522 DF32 (green) and Red chlorophyll catalase reductase labelled with Alexa Fluor 568 excited at a wavelength of $568 \mathrm{~nm}$ and detected using the emission filters 605 DF32 and 585 EFLP (red). Bar $=5 \mu \mathrm{m}$. A representative picture out of 11 transformed lines is presented

EDS5H to the EDS5 transporter region did not rescue SA accumulation (Fig. 7b). These results show that EDS5H does not function better in SA accumulation when the EDS5 N-terminus is present.

\section{Potential transport function of EDS5 and EDS5H}

Phylogenetic studies of the MATE-family of plants revealed that EDS5 and EDS5H are two transporters that are in the same subfamily of MATE-transporters as the Arabidopsis citrate transporters described recently [23,
24]. Therefore, EDS5 and EDS5H have been aligned in comparison to plant MATE transporters that have been shown to transport flavonoids (AtTT12) [18], alkaloids (NtMATE) [20] or other toxic molecules, such as polyvinylpyrrolidone and pyrrolidinone (AtAFL5) [25], as well as citrate transporters from monocots (barley [26], sorghum [27] and rice [28]) and Arabidopsis (AtFRD3 [29] and AtMATE [24]). This alignment shows that EDS5 and EDS5H form their own subgroup clustering together with the citrate transporters and not with the

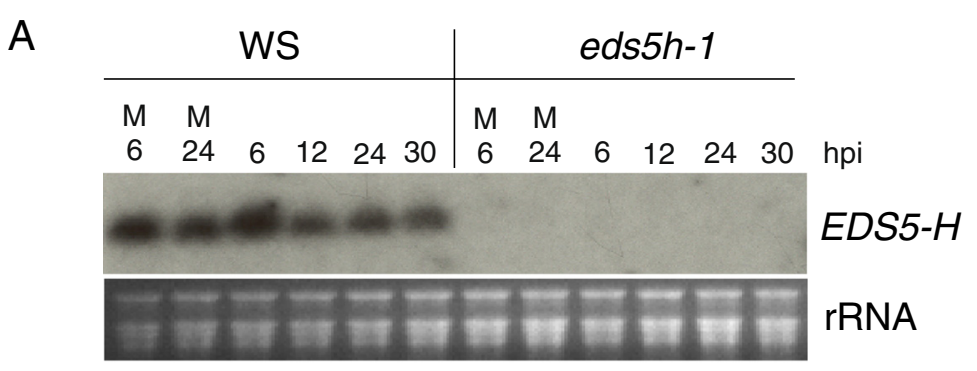

B

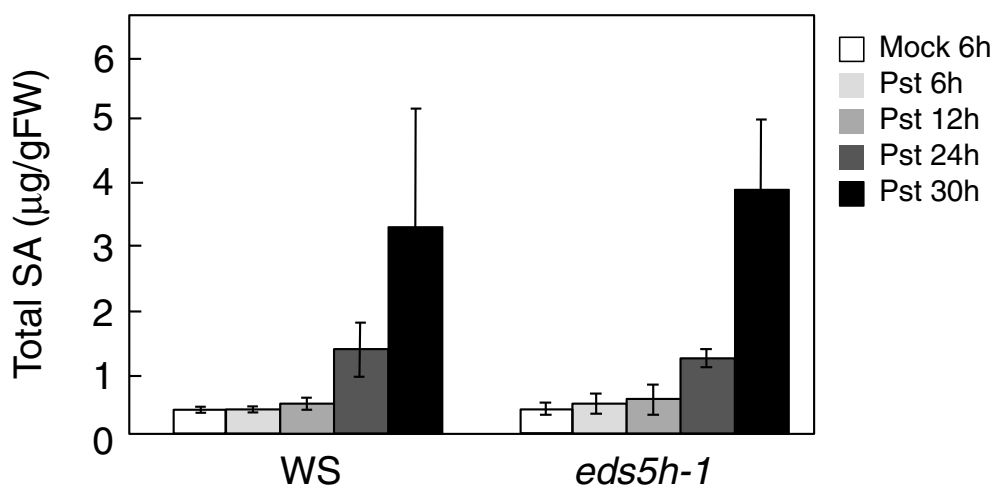

Fig. 5 Characterization of the eds5h-1 mutant. a) Expression analysis of the EDS5H. $10 \mathrm{mM} \mathrm{MgCl} 2$ (M) or P. syringae pv. tomato DC3000 were infiltrated into Ws and eds5h-1 leaves. Samples were taken at 6, 12, 24, and $30 \mathrm{~h}$ post-inoculation (hpi). Northern blots were hybridized with a gene-specific probe for EDS5H. Ethidium bromide staining of the RNA gel (rRNA) was used as control for the loading. The experiment was repeated 3 times with similar results. b) Accumulation of SA in plants infiltrated with $10 \mathrm{mM} \mathrm{MgCl}_{2}(\mathrm{M})$ or $P$. syringae pv. tomato DC3000 (Pst); leaves were harvested 6, 12, 24, and 30h hpi. For each time point $n=3( \pm S D)$, the experiment was repeated 3 times 


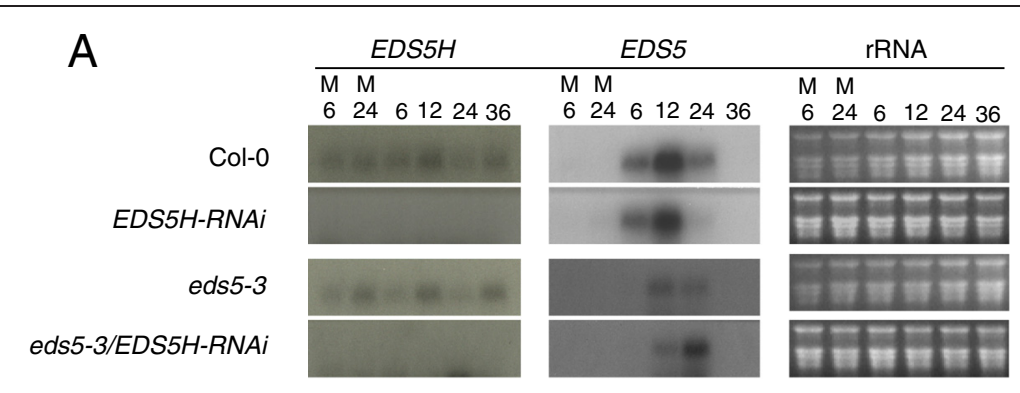

B

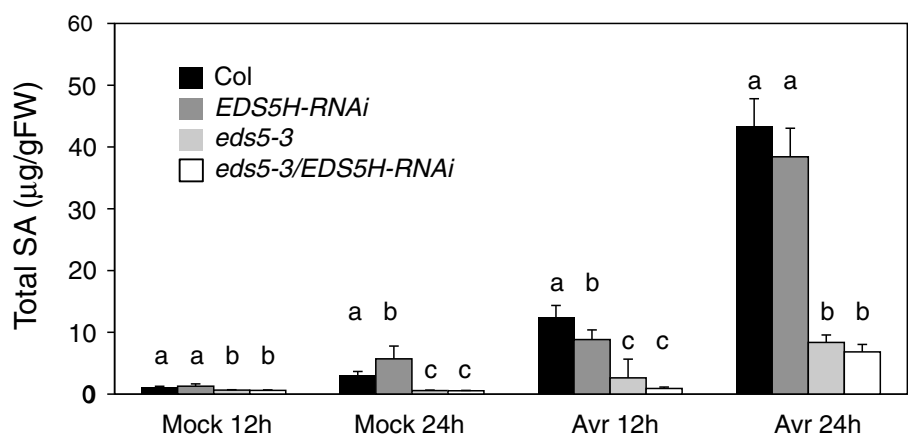

Fig. 6 Characterization of the EDS5H-RNAi mutant and eds5-3/EDS5H-RNAi double mutant. a) Expression analysis of EDS5H and EDS5 in different genotypes. Plants were inoculated with $10 \mathrm{mM} \mathrm{MgCl}$ (M) or P. syringae pv. tomato DC3000. Samples were taken at 6, 12, 24, and 36 hpi. Northern blots were hybridized with gene-specific probes for EDS5H and EDS5. Ethidium bromide staining of the RNA gel (rRNA) was used as loading control. Note that some EDS5 expression can be detected as eds5-3 is not a transcriptional null mutant. The experiment was repeated 3 times with similar results. b) Accumulation of SA in different genotypes. Plants were inoculated with $10 \mathrm{mM} \mathrm{MgCl}_{2}$ (Mock) or P. syringae pv. tomato DC3000 carrying avrRpt2 (Avr). Samples were taken 12 and 24 hpi. Different letters above each bar represent statistically significant differences. The mean comparison of total SA was analyzed using Duncan's multiple-range test (DMRT) with $p$-value $\leq 0.05$. For each time point $\mathrm{n}=4( \pm \mathrm{SD})$, the experiment was repeated 3 times

flavonoid/alkaloids/toxin transporters (Fig. 8). Since EDS5 was shown to transport SA, EDS5H might transport related phenolic compounds, but unlikely SA.

\section{Discussion}

A detailed characterization of the related MATEtransporter EDS5H was undertaken in order to obtain more information on its possible function relative to EDS5. The present studies confirm earlier results that EDS5 is only weakly expressed under non-inducing conditions and strongly after pathogen infection as reported for SA accumulation (Fig. 6a) [1]. The homologue EDS5H is constitutively expressed in all green tissues, independently of pathogen infection (Fig. 3). The fact that the eds5-3 mutant phenotype is clearly detectable under conditions where EDS5H is strongly expressed indicates that EDS5 and EDS5H may only have partially redundant functions.

The determination of the sub-cellular localization of EDS5H by fluorescence microscopy reveals that it is localized at the plastid where most of the SA is synthesized $[13,30]$. This is similar to EDS5 that was shown to localize within the chloroplast envelope and to function as a multidrug and toxin extrusion-like transporter in the export of SA from the chloroplast to the cytoplasm where it controls the innate immune response [13].

In order to clarify the functional relationship between EDS5 and EDS5H, T-DNA insertion mutants and RNAi lines where the $E D S 5 H$ transcript was knocked out have been isolated or produced in the Col-0 or in the eds5-3 mutant background. The analysis of all these lines clearly show that EDS5H is not involved in SA accumulation after pathogen or UV-light induction (Fig. 6). Two explanations may be proposed for why the eds5-3/eds $5 \mathrm{H}$ double mutants do not have less SA than eds5-3. Firstly, EDS5H could have a very low SA transport activity and therefore not reduce the SA content in the plastid. Secondly, the SA content that remains in the eds5-3 mutant is actually not produced in the plastid. Similarly, the single mutant ics 1 or the double mutant ics1/ics 2 contain a similarly low amount of SA indicating the existence of an alternative pathway for the production of SA [11]. Also, eds5-3 lines overexpressing EDS5H contain only slightly more SA independent of whether EDS5H was equipped with the N-terminus of EDS5 or not. These studies suggest that EDS5H has no, or at best a very 


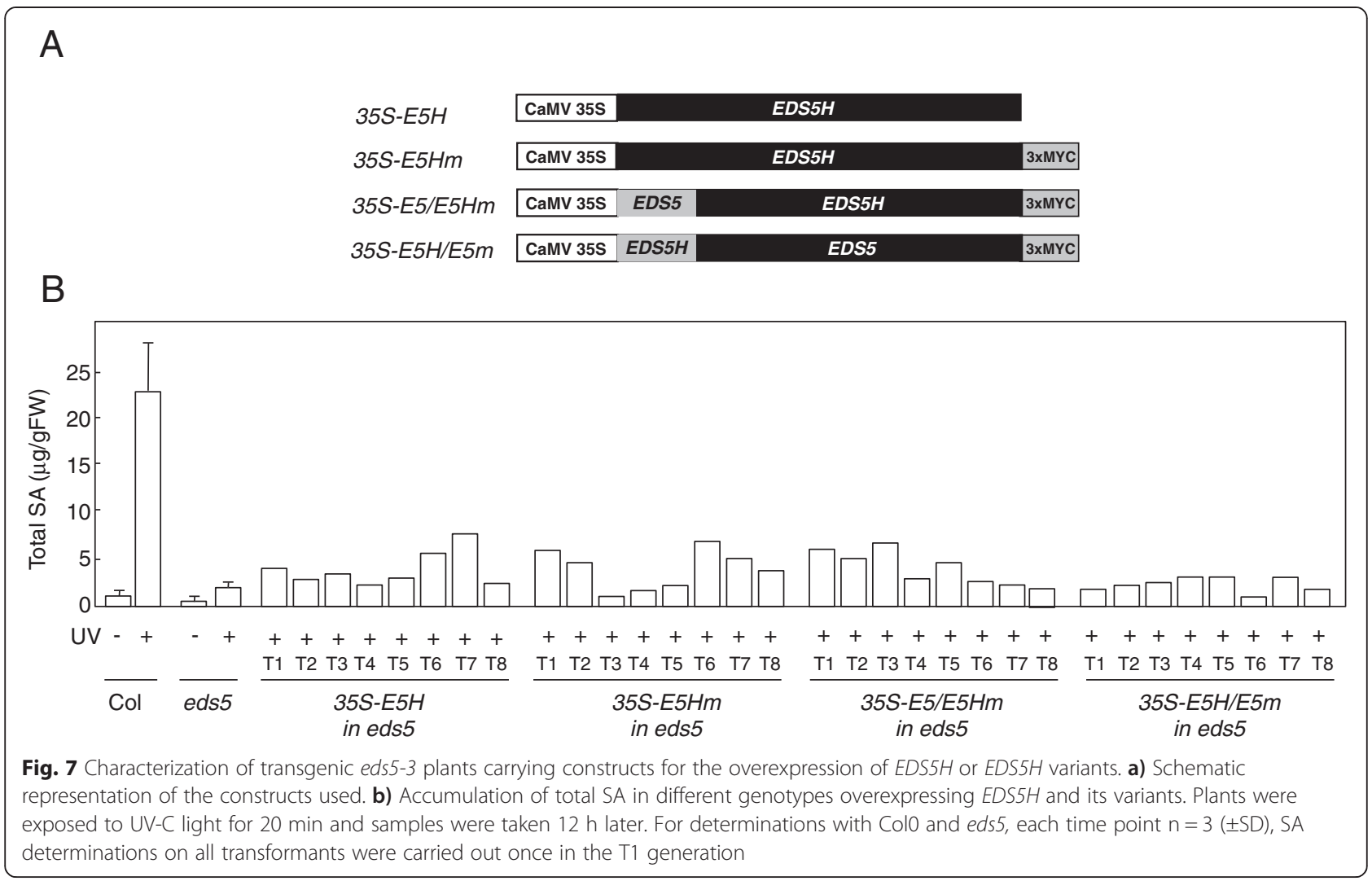

weak transport activity for SA and is not involved in resistance to $P$. syringae pv. tomato or Botrytis cinerea (unpublished data). Since EDS5H is closely related to EDS5 (Fig. 8) it is most likely involved in transporting related substances, for example other phenolic acids. The biochemical characterization of the transport activities of EDS5H will be exciting objectives for future work.

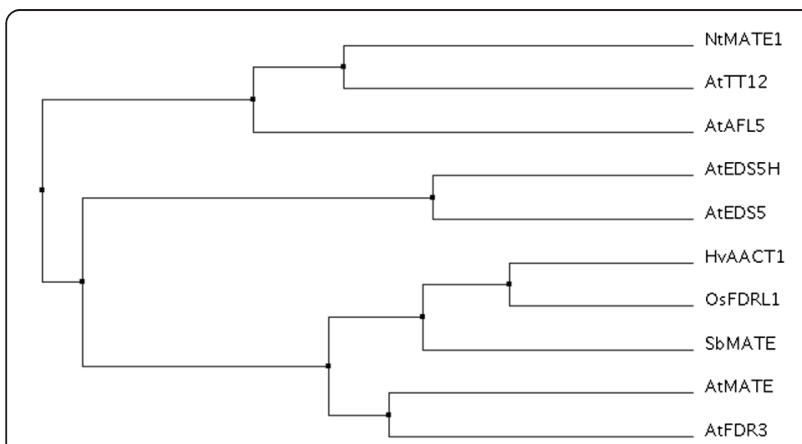

Fig. 8 Phylogenetic studies of the MATE-family of plants. EDS5 and EDS5H have been aligned in comparison to plant MATE shown to transport flavonoids (AtTT12) [18], alkaloids (NtMATE) [20] or molecules such as polyvinylpyrrolidone and pyrrolidinone (AtAFL5) [25], as well as citrate transporters from monocots (barley [26], sorghum [27] and rice [28]) and Arabidopsis (AtFRD3 [29] and AtMATE [24]). The dendrogram was established with the ClustalW program

\section{Conclusions}

Despite its homology to EDS5, EDS5H does not contribute to pathogen-induced SA accumulation like EDS5. The phylogenetic relatedness of EDS5H with EDS5 supports a function of EDS5H in the transport of phenolic substances.

\section{Materials and methods}

Growth conditions, bacterial inoculations and plant transformation

Arabidopsis accessions Columbia-0 (Col-0) and Wassilewskija (Ws) or transgenic plants were grown under either sterile or non-sterile conditions. Non-sterile plants were grown on a pasteurized soil mix of commercial potting soil:perlite (3:1), in a growth chamber at $22 \pm 2{ }^{\circ} \mathrm{C}$ under a 12-h photoperiod. Seed dormancy was broken by stratification at $4{ }^{\circ} \mathrm{C}$ for 3 days. For sterile cultures, seeds were surface sterilized by treating for $15 \mathrm{~min}$ in $2.5 \%(\mathrm{v} / \mathrm{v})$ commercial bleach containing $0.05 \%$ Triton X-100 with continuous agitation and rinsed four times with sterile distilled water. Seeds were placed on solid medium consisting of $1 / 2$ MS (Murashige and Skoog basal medium, Sigma) with $1 \%$ sucrose, $0.1 \%$ vitamins (Murashige and Skoog, Sigma). P. syringae pv tomato with or without avrRpt 2 was cultured at $28{ }^{\circ} \mathrm{C}$ and $220 \mathrm{rpm}$ in LuriaBertani medium containing $30 \mu \mathrm{g} / \mathrm{ml}$ rifampicin and 
$50 \mu \mathrm{g} / \mathrm{ml}$ kanamycin. Four-week-old plants were syringe-inoculated with a suspension of $\sim 10^{5}$ colonyforming units per $\mathrm{ml}$ in $10 \mathrm{mM} \mathrm{MgCl} 2$ and mock control plants with $10 \mathrm{mM} \mathrm{MgCl} 2$.

Arabidopsis was transformed by the floral dip method [31]. A. tumefaciens strain GV3101, transformed by heat shock with the construct of interest, was grown at $28^{\circ} \mathrm{C}$ in LB medium with an appropriate antibiotic. Transgenic plants were selected on $1 / 2$ MS plates, containing $30 \mu \mathrm{g} / \mathrm{ml}$ hygromycin.

\section{Cloning of EDS5H and sequence analysis}

cDNA of EDS5H (At2g21340) was amplified from Col-0 plants using the Access RT-PCR system from Promega. The 5' end of the cDNA was determined by RT-PCR using primers located 75 (75UpStream), and 150 (150UpStream) bp upstream of the predicted ATG on the annotated genomic sequence (for these and all other primers indicated below, see sequences in Additional file 1: Figure S1). The 3' end of the cDNA was determined by RTPCR using primers located 50 (50DownStream), 100 (100DownStream), 200 (200DownStream), 250 (250DownStream), and 300 (300DownStream) bp downstream of the stop codon of the annotated genomic sequence. RT-PCR products were cloned into pGEM-T Easy Vector (Promega) for sequencing.

\section{Isolation of RNA and DNA, transcript analysis}

For RNA gel blot analysis, RNA was isolated as previously described [4]. For qPCR, RNA was isolated using the Qiagen RNA easy kit including the recommended DNAase treatment. Genomic DNA was isolated using a protocol modified from [32]. Briefly, a leaf was extracted with buffer (0.2 M Tris- $\mathrm{HCl} \mathrm{pH}$ 9.0, 0.4 M LiCl, $25 \mathrm{mM}$ EDTA, $1 \%$ SDS). The extracted tissue was centrifuged and the DNA was precipitated with isopropanol. The airdried pellet was resuspended in TE $(10 \mathrm{mM}$ Tris pH 8.0, 1 mM EDTA).

For RNA gel blot analysis, total RNA $(10 \mu \mathrm{g})$ was separated in formaldehyde-agarose $(1 \%)$ gels, transferred to a Nylon membrane (Hybond-N, Amersham Biosciences, UK) and crosslinked by UV-light. Hybridization was carried out in hybridization buffer $\left(0.5 \mathrm{M} \mathrm{NaHPO}_{4} \mathrm{pH} 7.2\right.$, $7 \%$ SDS, 1 mM EDTA, $1 \%$ BSA) at $65^{\circ} \mathrm{C}$. The membrane was washed twice with $2 \mathrm{X}$ SSC containing $0.1 \%$ SDS and twice with $0.2 \mathrm{XSC}$ containing $0.1 \%$ SDS at $65{ }^{\circ} \mathrm{C}$, before exposing to X-Omat or Bio Max film (Kodak).

\section{Isolation of mutants carrying a T-DNA in the EDS5H region}

Plants carrying a T-DNA fragment in the region of exon 1 and exon 10, called eds5h-1 and eds5H-10, respectively, were screened by PCR www.biotech.wisc.edu/ NewServicesAndResearch/Arabidopsis. eds5H-1 plants were isolated by using the forward primer JL-202, which is present on the left border of T-DNA region (JL-202) and the reverse primer GSP1. The homozygous line was selected by the absence of the wild-type fragment using GSP2 and GSP1 as forward and reverse primers, respectively. eds 5 h-10 plants were selected by using JL-202 as forward primer and GSP3 as reverse primer. A homozygous line was identified by the absence of the wild-type fragment by using GSP4 and GSP3 as forward and reverse primers, respectively.

\section{Construction of the plasmid used for RNAi}

A fragment of about $350 \mathrm{bp}$ of the non-conserved region of EDS5H was amplified by RT-PCR and cloned into plasmid pHANNIBAL [22] in sense and anti-sense orientation. The sense strand was designed to include $\mathrm{XhoI}$ and $\mathrm{Kp} n \mathrm{I}$ as cloning sites (forward primer: $\mathrm{E} 5 \mathrm{H}-$ sens-For; reverse primer: E5H-sens-Rev) and the antisense strand was designed to include $\mathrm{BamHI}$ and $\mathrm{ClaI}$ as cloning sites (forward primer: E5H-anti-For; reverse primer: $\mathrm{E} 5 \mathrm{H}$-anti-Rev). The construct was cloned into the binary vector PART27 using the NotI sites [33].

\section{Construction of promoter:GUS and cMyc-tagged constructs}

Three fragments of the promoter region of $E D S 5 H$ were amplified using the High Fidelity Kit (Roche) with genespecific primers designed to introduce EcoRI at the 5', ends and NcoI sites at the 3' ends of the fragments. The forward primers used for the amplification of EDS5H promoter fragments were: Pro500F, Pro1000F and Pro2000F resulting in fragments of $546 \mathrm{bp}, 1057$ and $1984 \mathrm{bp}$, respectively. The reverse primer for the EDS5H promoter fragments was ProE5H-R. The PCR fragments were cloned into the plasmid pCAMBIA 1303 (www.CAMBIA.org) from which the fragments of the promoter CaMV $35 \mathrm{~S}$ and LacZ alpha had been removed.

The ORF of EDS5H was amplified by High-fidelity RT-PCR using the Omniscript RT-PCR system (Qiagen) and the forward primer Nco-E5H introducing a NcoI site as well the reverse primer E5H-Mycs introducing a triple cMyc-tag and the restriction sites SmaI and NdeI. The PCR fragment was cloned into pGEM-T Easy Vector (Promega) in the NcoI and NdeI sites. The clone was sequenced and the NcoI-SmaI fragment that included EDS5H::3xcMyc-tagg was then cloned into pCAMBIA 1304 (www.CAMBIA.org) from which the GUS and GFP genes had been removed.

\section{Construction of plasmid for overexpressing of EDS5H}

The cDNA of EDS5H was amplified by RT-PCR using the forward primer E5H-For and the reverse primer E5H-Rev and was sub-cloned into pGEM-T Easy Vector (Promega). The cDNA was digested with EcoRI, blunted, 
and cloned into pART7 [33]. Clones were selected based on the correct orientation of the cDNA between the CaMV35S promoter and OCS terminator. The cassette of CaMV35S::EDS5H::OCS was digested with NotI and cloned into the binary vector pART27 [33].

\section{Constructions of plasmids for protein domain swapping}

The constructs for the peptide-swapping experiments were designed to include the triple cMyc-epitope tag (3xcMyc-tag). Therefore, a fragment of 3xcMyc-tag was amplified using the synthesized oligonucleotide E5HMycs as template. The PCR fragment included a Not I site at 5'-end and a SmaI and NdeI sites at 3'-end and 4 alanine residues upstream of cMyc-tag (forward primer: For-Ala-Myc; reverse primer: Rev-Myc-PCS). The $3 x c M y c-t a g$ was cloned into pGEM-T Easy vector at corresponding sites.

$E D S 5$ and $E D S 5 H$ genes were swapped at the NdeI site of EDS5 (281 bp downstream of ATG). The NdeI site was introduced into $E D S 5 H$ by changing of a nucleotide $\mathrm{T}$ to A (336 bp downstream of ATG) that caused no change in amino acid residues. Therefore, the Nterminus of each protein was designed to include $\mathrm{NcoI}$ and NdeI as cloning sites. The fragments were amplified by RT-PCR using forward primer Nco-EDS5, reverse primer EDS5-Nde for EDS5, and forward primer Nco-E5H, reverse primer $\mathrm{E} 5 \mathrm{H}-\mathrm{Nde}$ for $\mathrm{EDS} 5 \mathrm{H}$. The $\mathrm{C}$-terminus of each protein was designed to include NdeI and NotI as cloning sites. The fragments were amplified using forward primer Nde-eds5, reverse primer EDS5-Rev for EDS5, and forward primer Nde-e5h, reverse primer E5H-Swap-Rev for EDS5H. The swapped domains of either EDS5::EDS5H or EDS5H::EDS5 were constructed in pBluescript (Stratagene).

The constructs were placed upstream of 3xcMyc-tag with NcoI and NotI sites. The cassettes of the swapped peptide domains and 3xcMyc-tag were then cloned into pCAMBIA 1304 (www.CAMBIA.org). The GFP and GUS reporter genes of the binary vector were replaced with either EDS5:::EDS5H::3xcMyc-tag or EDS5H::EDS5::3xcMyctag cassettes at $\mathrm{NcoI}$ and PmlI sites.

\section{Immunolabelling and fluorescence microscopy}

For immunolabelling, plant tissue was prepared according to the protocol described in [34]. The PEG-embedded tissue was sectioned into $4-5 \mu \mathrm{m}$-thick slices and placed on poly-L-lysine-covered slides. The slides were incubated in PBS for $10 \mathrm{~min}$ in order to remove the PEG from the section. Each slide was blocked for free aldehyde by incubation with $0.1 \mathrm{M} \mathrm{NH}_{4} \mathrm{Cl}$ (in PBS) for $5 \mathrm{~min}$, and washed for 5 min with PBS. The unspecific binding sites were blocked by incubation with $5 \%$ BSA (in PBS) for $30 \mathrm{~min}$. Slides were then incubated overnight with primary antibody solution (mouse anti-cMyc diluted in $5 \%$ BSA/PBS in ratio
1:500) at $4{ }^{\circ} \mathrm{C}$, washed three times with $0.1 \%$ BSA (in PBS) for 10 min each, and once with $1 \%$ BSA (in PBS) for $10 \mathrm{~min}$. The secondary antibody used was goat antimouse-IgG conjugated with Alexa Fluor 488 or goat antirabbit-IgG conjugated with Alexa Fluor 568, diluted 1:500 in $5 \%$ BSA (in PBS). Slides were placed in a humid chamber, incubated with secondary antibody for $2 \mathrm{~h}$ at $37^{\circ} \mathrm{C}$. After washing with PBS (4x10 min, samples were kept at $4{ }^{\circ} \mathrm{C}$ in the dark. Fluorescent signals were recorded with a BioRad MCR 1024 Kripton-Argon confocal microscope using an excitation wavelength of $488 \mathrm{~nm}$ with an emission filter 522 DF32, or an excitation wavelength of $568 \mathrm{~nm}$ with emission filters 605 DF32 and 585 EFLP.

\section{GUS-assays and determination of SA}

GUS assays and the determination of free and conjugated SA were performed as described previously $[4,35]$.

\section{Additional file}

Additional file 1: List of primers.

\section{Competing interests}

The authors declare that they have no competing interests.

\section{Authors' contributions}

NP carried out the molecular genetic studies, participated in the sequence alignment. SC contributed to the localization studies. AB carried out the SA analyses. CN designed the study, performed the statistical analysis, drafted the manuscript. JPM drafted the final version of manuscript. All authors read and approved the final manuscript.

\section{Acknowledgements}

We would like to thank Linda Grainger and Martine Schorderet for excellent technical assistance. This work was supported by grants from the Swiss

National Science Foundation to Jean-Pierre Métraux and Christiane Nawrath.

\section{Author details}

${ }^{1}$ Department of Biology, University of Fribourg, 1700 Fribourg, Switzerland. ${ }^{2}$ Faculty of Agricultural Technology, King Mongkut's Institute of Technology Ladkrabang, Chalongkrung Rd., Ladkrabang, 10520 Bangkok, Thailand. ${ }^{3}$ Department of Plant Molecular Biology, University of Lausanne, 1015 Lausanne, Switzerland.

Received: 17 January 2015 Accepted: 1 May 2015 Published online: 09 June 2015

\section{References}

1. Nawrath C, Metraux JP. Salicylic acid induction-deficient mutants of Arabidopsis express PR-2 and PR-5 and accumulate high levels of camalexin after pathogen inoculation. Plant Cell. 1999;11:1393-404.

2. Vlot AC, Dempsey DMA, Klessig DF. Salicylic acid, a multifaceted hormone to combat disease. Annu Rev Phytopathol. 2009;47:177-206.

3. Yan S, Dong X. Perception of the plant immune signal salicylic acid. Curr Opin Plant Biol. 2014;20:64-8.

4. Nawrath C, Heck S, Parinthawong N, Metraux JP. EDS5, an essential component of salicylic acid-dependent signaling for disease resistance in Arabidopsis, is a member of the MATE transporter family. Plant Cell. 2002;14:275-86.

5. Wildermuth MC, Dewdney J, Wu G, Ausubel FM. Isochorismate synthase is required to synthesize salicylic acid for plant defence. Nature. 2001;417:562-5.

6. Strawn MA, Marr SK, Inoue K, Inada N, Zubieta C, Wildermuth MC Arabidopsis isochorismate synthase functional in pathogen-induced salicylate 
biosynthesis exhibits properties consistent with a role in diverse stress responses. J Biol Chem. 2007;282:5919-33.

7. Ogawa D, Nakajima N, Tamaoki M, Aono M, Kubo A, Kamada H, et al. Salicylic acid accumulation is controlled by feedback inhibition in ozone-exposed Arabidopsis. Plant Cell Physiol. 2006;47:S233.

8. Verberne MC, Sansuk K, Bol JF, Linthorst HJM, Verpoorte R. Vitamin K-1 accumulation in tobacco plants overexpressing bacterial genes involved in the biosynthesis of salicylic acid. J Biotechnol. 2007;128:72-9.

9. Uppalapati SR, Ishiga Y, Wangdi T, Kunkel BN, Anand A, Mysore KS, et al. The phytotoxin coronatine contributes to pathogen fitness and is required for suppression of salicylic acid accumulation in tomato inoculated with Pseudomonas syringae pv. tomato DC3000. Mol Plant Microbe Interact. 2007;20:955-65.

10. Catinot J, Buchala A, Abou-Mansour E, Métraux J-P. Salicylic acid production in response to biotic and abiotic stress depends on isochorismate in Nicotiana benthamiana. FEBS Lett. 2008:582:473-8.

11. Garcion C, Lohmann A, Lamodiere E, Catinot J, Buchala A, Doermann P, et al. Characterization and biological function of the ISOCHORISMATE SYNTHASE2 gene of Arabidopsis. Plant Physiol. 2008;147:1279-87.

12. Gaille $C$, Kast P, Haas D. Salicylate biosynthesis in Pseudomonas aeruginosa. Purification and characterization of PCHB, a novel bifunctional enzyme displaying isochorismate pyruvate-lyase and chorismate mutase activities. J Biol Chem. 2002;277:21768-75.

13. Serrano M, Wang BJ, Aryal B, Garcion C, Abou-Mansour E, Heck S, et al. Export of salicylic acid from the chloroplast requires the Multidrug and Toxin Extrusion-Like transporter EDS5. Plant Physiol. 2013;162:1815-21.

14. Yamasaki K, Motomura Y, Yagi Y, Nomura H, Kikuchi S, Nakai M, et al. Chloroplast envelope localization of EDS5, an essential factor for salicylic acid biosynthesis in Arabidopsis thaliana. Plant Signal Behav. 2013;8:e23603.

15. Kuroda T, Tsuchiya T. Multidrug efflux transporters in the MATE family. Biochim Biophys Acta. 2009;1794:763-8.

16. Moriyama $\mathrm{Y}$, Hiasa M, Matsumoto $\mathrm{T}$, Omote H. Multidrug and toxic compound extrusion (MATE)-type proteins as anchor transporters for the excretion of metabolic waste products and xenobiotics. Xenobiotica. 2008;38:1107-18.

17. Debeaujon I, Peeters AJM, Leon-Kloosterziel KM, Koornneef M. The TRANSPARENT TESTA12 gene of Arabidopsis encodes a multidrug secondary transporter-like protein required for flavonoid sequestration in vacuoles of the seed coat endothelium. Plant Cell. 2001;13:853-71.

18. Marinova K, Pourcel L, Weder B, Schwarz M, Barron D, Routaboul J-M, et al. The Arabidopsis MATE transporter $T 12$ acts as a vacuolar flavonoid/H + -antiporter active in proanthocyanidin-accumulating cells of the seed coat. Plant Cell. 2007;19:2023-38.

19. Morita M, Shitan N, Sawada K, Van Montagu MCE, Inzé D, Rischer H, et al. Vacuolar transport of nicotine is mediated by a multidrug and toxic compound extrusion (MATE) transporter in Nicotiana tabacum. Proc Nat Acad Sci. 2009;106:2447-52

20. Shoji T, Inai K, Yazaki Y, Sato Y, Takase H, Shitan N, et al. Multidrug and toxic compound extrusion-type transporters implicated in vacuolar sequestration of nicotine in tobacco roots. Plant Physiol. 2009;149:708-18.

21. Rodoni S, Vicentini F, Schellenberg M, Matile P, Hortensteiner S. Partial purification and characterization of red chlorophyll catabolite reductase, a stroma protein involved in chlorophyll breakdown. Plant Physiol. 1997;115:677-82.

22. Helliwell C, Waterhouse P. Constructs and methods for high-throughput gene silencing in plants. Methods. 2003;30:289-95.

23. Li LG, He ZY, Pandey GK, Tsuchiya T, Luan S. Functional cloning and characterization of a plant efflux carrier for multidrug and heavy metal detoxification. J Biol Chem. 2002;277:5360-8.

24. Liu J, Magalhaes J, Shaff J, Kochian L. Aluminum-activated citrate and malate transporters from the MATE and ALMT families function independently to confer Arabidopsis aluminum tolerance. Plant J. 2009;57:389-99.

25. Diener AC, Gaxiola RA, Fink GR. Arabidopsis ALF5, a multidrug efflux transporter gene family member, confers resistance to toxins. Plant Cell. 2001;13:1625-37.

26. Furukawa J, Yamaji N, Wang H, Mitani N, Murata Y, Sato K, et al. An aluminum-activated citrate transporter in barley. Plant Cell Physiol. 2007:48:1081-91

27. Magalhaes J, Liu J, Guimaraes C, Lana U, Alves V, Wang Y, et al. A gene in the multidrug and toxic compound extrusion (MATE) family confers aluminum tolerance in sorghum. Nat Genet. 2007;39:1156-61.
28. Yokosho K, Yamaji N, Ueno D, Mitani N, Ma JF. OsFRDL1 is a citrate transporter required for efficient translocation of iron in rice. Plant Physiol. 2009;149:297-305.

29. Durrett T, Gassmann W, Rogers E. The FRD3-mediated efflux of citrate into the root vasculature is necessary for efficient iron translocation. Plant Physiol. 2007;144:197-205.

30. Fragnière C, Serrano M, Abou-Mansour E, Metraux J-P, L'Haridon F. Salicylic acid and its location in response to biotic and abiotic stress. FEBS Lett. 2011;585:1847-52.

31. Clough SJ, Bent AF. Floral dip: a simplified method for Agrobacteriummediated transformation of Arabidopsis thaliana. Plant J. 1998;16:735-43.

32. Sussman MR, Amasino RM, Young JC, Krysan PJ, Austin-Phillips S. The Arabidopsis knockout facility at the University of Wisconsin-Madison. Plant Physiol. 2000;124:1465-1467.

33. Gleave AP. A versatile binary vector system with a T-DNA organizationalstructure conducive to efficient Integration of cloned DNA into the plant Genome. Plant Mol Biol. 1992;20:1203-7.

34. Vanlammeren AAM, Keijzer CJ, Willemse MTM, Kieft H. Structure and function of the microtubular cytoskeleton during pollen development in Gasteria verrucosa (Mill) H Duval. Planta. 1985;165:1-11.

35. Ribot $C$, Wang $Y$, Poirier $Y$. Expression analyses of three members of the AtPHO1 family reveal differential interactions between signaling pathways involved in phosphate deficiency and the responses to auxin, cytokinin, and abscisic acid. Planta. 2008;227:1025-36.

\section{Submit your next manuscript to BioMed Central and take full advantage of:}

- Convenient online submission

- Thorough peer review

- No space constraints or color figure charges

- Immediate publication on acceptance

- Inclusion in PubMed, CAS, Scopus and Google Scholar

- Research which is freely available for redistribution 